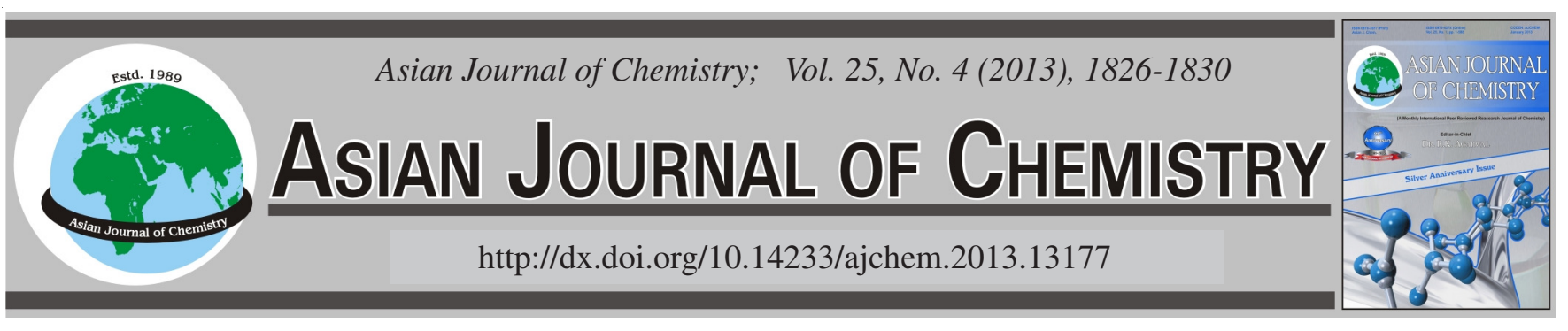

\title{
Intraparticle Mass Transfer Model of Ethylene Polymerization Catalyzed by Supported Metallocene
}

\author{
KAI HuANG ${ }^{1,2, *}$ and RuIJIN XIE ${ }^{1}$
}

${ }^{1}$ School of Chemistry and Chemical Engineering, Southeast University, Nanjing 211189, P.R. China

${ }^{2}$ Changzhou Institute, Southeast University, Changzhou 213164, P.R. China

*Corresponding author: Tel: +86 139 13871802; E-mail: huangk@seu.edu.cn

(Received: 5 December 2011;

Accepted: 3 October 2012)

AJC-12228

The slurry ethylene polymerization catalyzed with a silica-supported metallocene catalyst was simulated based on a modified multigrain
model. The modified model combined with the intrinsic reaction investigation was used to analyze the catalyst fragmentation and the
polyethylene intraparticle mass transfer. The model can also be used to calculate the monomer concentration and the evolution of the
macroparticle size. Furthermore, the model can also be used to predict the changes of the particle porosity and the monomer concentration
distribution. The results show that the intraparticle mass transfer resistance has great effect on the polymerization and the catalyst
fragmentation will take place shell by shell from the surface to the center after $200 \mathrm{~s}$ of the polymerization time.

Key Words: Metallocene catalyst, Ethylene polymerization, Polyethylene, Single particle mass transfer model, Catalyst fragmentation.

ᄂ - - - - - - - - - - - - - - - - - - - - - - - - - - -

\section{INTRODUCTION}

Slurry phase polymerization of ethylene catalyzed by supported metallocene catalyst was studied in recent years ${ }^{1-17}$. Various models at the mesoscale level for slurry phase polymerization of olefins catalyzed by metallocene catalyst have been established.

Begley $^{2}$ proposed the first and simplest model in 1966, which was generally known as 'solid core model'. The model was based on a spherical catalyst particle with a spherical shell of polymer growing around it. The polymeric core model was derived by Schmeal and Street $^{3}$, in the model, expansion of the catalytic pellets was considered but the diameter of the catalytic pellet remained stable and only the thickness of the polymer shell grew. The increasing particle size was taken into consideration in the polymeric flow model ${ }^{4}$ during the polymerization as well as the drift of the active sites.

The experimental evidence showed that the catalytic particles were broken into many small fragments. The multigrain model $l^{5}$ was developed to simulate the complex and realistic polymerizing particles. This model assumed that macroparticle consisted of many small microparticles (the fragments) and each microparticle act like a 'solid core' pellet. This last model was derived for conventional Ziegler-Natta catalysts and only a complete fragmented particle was considered.

As realizing that the multigrain model could not explain experimental data, Bonini et al. ${ }^{6}$ developed a partially particle growth model, based on a shell by shell fragmentation hypothesis (gradual break-up from the outside to the inside of the particle) and a final multigrain structure of the particle. In this way the pellet is divided into two parts: a fragmented (that behaves exactly like in the multigrain model) and an unfragmented one.

The above model could explain the acceleration of reaction rate, but fail to predict the initial peak, which can be shown in the reaction rate curve. Alexiadis et al. ${ }^{7,8}$ proposed a model by using Bonini's example, but with the addition of a further part regarding the unfragmentation core region and assumed that the monomer move fast in fragmentation region than in the solid core. It should be pointed out that Alexiadis et al. ${ }^{7,8}$ assumed that the particle kept constant void fraction under reaction, but porosity of the particle varied with reaction. Estenoz and Chiovetta ${ }^{9}$ put forward a model on the basis of the multigrain model, by taking account the variable void fraction. However, Estenoz's model neglected the difference of mass transfer between fragmentation region and unfragmentation region. It is clear that this idea is in contradiction with the experimental data. The simulation results of the polymerization rate were compared with the experiment data by Alexiadis and Andes ${ }^{8}$.

In this paper, a single particle model, which combines some of the properties of Alexiadis's model and Estenoz's model, is proposed. The model, which analyzes the catalyst fragmentation and the polyethylene intraparticle mass transfer 
by coupling with the intrinsic reaction, can be used to calculate the monomer concentration and the evolution of the macroparticle size.

Polymer particle model: Based on the previous references, the polyethylene intraparticle heat transfer is very little and can be neglected in slurry phase polymerization. An activated porous catalyst particle, which size is $5-100 \mu \mathrm{m}$, is composed of microparticle crystallites of size in the range of $0.001-0.5 \mu \mathrm{m}$. At the beginning of polymerization, the monomer diffuses through the interstices between crystallites and the polymerization occurs on the surface of the catalyst microparticle crystallites. The polymer accumulates around the catalyst primary crystallites and this accumulation forces the crystallites further broken up. Thus the polymer particle grows larger and larger during polymerization. As polymerization goes by, the original catalyst particle breaks up into a much larger number of microparticle shell-by-shells from the surface to the center, as shown in the Fig. 1.

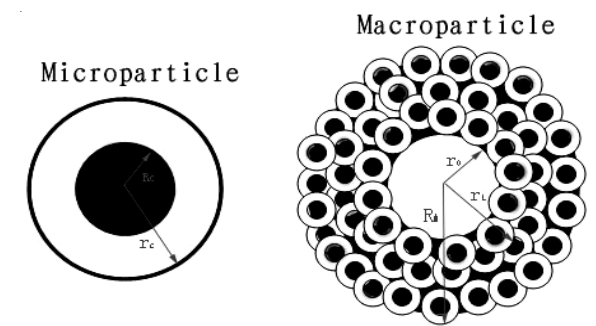

Fig. 1. Schematic representation of model for polyethylene particle growth

In the modified multigrain model, it is assumed that (i) the catalyst particle is spherical and microparticles are the same. (ii) The catalyst particle is divided into several concentric spherical layers, which is also divided into fragmentation zone and unfragmentation zone. (iii) Every layer in the catalyst particle is the uniform body and the monomer, active sites, polymers are dispersed in it. (iv) The catalyst is activated instantly and catalyst is not dead activated.

Single particle model: To simulate particle growth and morphology, the radial profile of monomer concentration in a polymer particle should be developed. According to the mass and energy balance, the equations of the monomer for macroparticle are given as follow.

Fragmentation region ${ }^{8}$ :

$$
\begin{gathered}
\frac{\partial \mathrm{M}}{\partial \mathrm{t}}=\frac{1}{\mathrm{r}_{1}^{2}} \frac{\partial}{\partial \mathrm{r}_{1}}\left(\mathrm{D}_{1} \mathrm{r}_{1}^{2} \frac{\partial \mathrm{M}}{\partial \mathrm{t}}\right)-\frac{\mathrm{R}_{\mathrm{p}}}{\varepsilon} \\
\frac{\partial \mathrm{T}_{1}}{\partial \mathrm{t}}=\frac{1}{\mathrm{r}_{1}^{2}} \frac{\partial}{\partial \mathrm{r}_{1}}\left(\frac{\mathrm{k}_{\mathrm{e}}}{\rho_{\mathrm{M}} \mathrm{c}_{\mathrm{p}}} \mathrm{r}_{1}^{2} \frac{\partial \mathrm{T}_{1}}{\partial \mathrm{r}_{1}}\right)-\frac{\Delta \mathrm{H}}{\rho_{\mathrm{M}} \mathrm{c}_{\mathrm{p}}} \mathrm{R}_{\mathrm{p}}
\end{gathered}
$$

The initial and boundary conditions of eqns. 3 and 4 are given by:

$$
\begin{gathered}
\frac{\partial \mathrm{M}}{\partial \mathrm{r}_{1}}=\mathrm{k}_{\mathrm{s}}\left(\mathrm{M}_{\mathrm{B}}-\mathrm{M}\right) \frac{\partial \mathrm{T}}{\mathrm{k}_{\mathrm{M}}}=\frac{\mathrm{h}}{\mathrm{k}_{\mathrm{M}}}\left(\mathrm{T}_{\mathrm{B}}-\mathrm{T}\right) \\
\mathrm{r}_{1}=\mathrm{R}_{\mathrm{M}} \mathrm{t}=\mathrm{t}
\end{gathered}
$$

Unfragmentation region ${ }^{7}$ :

$$
\frac{\partial \mathrm{M}}{\partial \mathrm{t}}=\frac{1}{\mathrm{r}_{1}^{2}} \frac{\partial}{\partial \mathrm{r}_{1}}\left(\mathrm{X}_{\mathrm{D}} \mathrm{D}_{1} \mathrm{r}_{1}^{2} \frac{\partial \mathrm{M}}{\partial \mathrm{t}}\right)-\frac{\mathrm{R}_{\mathrm{p}}}{\varepsilon}
$$

$$
\frac{\partial \mathrm{T}_{1}}{\partial \mathrm{t}}=\frac{1}{\mathrm{r}_{1}^{2}} \frac{\partial}{\partial \mathrm{r}_{1}}\left(\frac{\mathrm{k}_{\mathrm{e}}}{\rho_{\mathrm{M}_{\mathrm{p}}} \mathrm{c}_{1}} \mathrm{r}_{1}^{2} \frac{\partial \mathrm{T}_{1}}{\partial \mathrm{r}_{1}}\right)-\frac{\Delta \mathrm{H}}{\rho_{\mathrm{M}} \mathrm{c}_{\mathrm{p}}} \mathrm{R}_{\mathrm{p}}
$$

The initial and boundary conditions of eqn. 7 and 8 are given by:

$$
\begin{gathered}
\frac{\partial \mathrm{M}}{\partial \mathrm{r}_{1}}\left|\xi=\mathrm{X}_{\mathrm{D}} \frac{\partial \mathrm{M}}{\partial \mathrm{r}_{1}}, \frac{\partial \mathrm{T}}{\partial \mathrm{r}_{1}}\right| \xi=\frac{\partial \mathrm{T}}{\partial \mathrm{r}_{1}} \\
\frac{\partial \mathrm{M}}{\partial \mathrm{t}}=0 \quad \frac{\partial \mathrm{T}}{\partial \mathrm{t}}=0 \quad \mathrm{r}_{1}=0 \quad \mathrm{t}=\mathrm{t}
\end{gathered}
$$

This parameter ${ }^{7} \mathrm{X}_{\mathrm{D}}$ is defined as the ratio between the monomer diffusion constant in the macroparticle before and after fragmentation.

Volume balance over microparticle: The volume balance over the microparticle can be given by ${ }^{18,19}$ :

$$
\frac{\mathrm{dV}}{\mathrm{dt}}=4 \pi \mathrm{R}_{\mathrm{c}} \frac{\mathrm{dR}}{\mathrm{dt}}=\frac{4}{3} \pi \mathrm{R}_{\mathrm{c}} \mathrm{k}(\mathrm{T}) \mathrm{M}_{\mathrm{c}} \frac{\mathrm{M}_{\mathrm{n}}}{\rho_{\mathrm{p}}}
$$

The parameter $\mathrm{C}$ is defined as growth factor of single

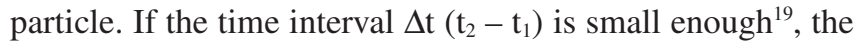
time-dependent growth factor, $\mathrm{C}(\mathrm{t})$, can be calculated approximately by:

$$
C\left(t_{2}\right)=\left[C\left(t_{1}\right)^{3}+\frac{k(T) M_{n}\left(t_{2}-t_{1}\right)}{\rho_{p} R_{c}} \times \frac{\left(M_{c}\left(t_{1}\right)+M_{c}\left(t_{2}\right)\right)}{2}\right]^{1 / 3}
$$

The quasi steady-state approximation (QSSA) is used for the microparticle mass balance and the temperature gradient can be neglected based on the previous research. By using the QSSA presented in Hutchinson el al. ${ }^{18}$, the mass balance on the catalyst surface can be obtained as follow:

$$
\mathrm{M}_{\mathrm{c}}=\frac{\alpha \mathrm{M}}{1+\frac{1}{3} \frac{\beta^{2}(\mathrm{C}-1)}{\mathrm{C}}}
$$

where $\beta$ denotes a Thiele modulus, $\beta=R_{c} \sqrt{\frac{k}{D_{s}}}, C$ denotes the microparticle growth factor and a denotes the amorphousphase volume fraction within the polymer layer, the simulation results show that a very small time step is necessary to track the rapid change in the particle.

Particle growth and grid updating: As the time interval $\Delta t\left(t_{2}-t_{1}\right)$ increases, the void fraction and position of each layer must be updated, which is ignored by previous workers ${ }^{1,6,8-10}$. For considering this change, the following equation can be derived by the workof Fink et al. ${ }^{10}$ and our work is different with Ref. ${ }^{7}$.

In unfragmentation region ${ }^{9}$

$$
\begin{gathered}
\varepsilon\left(\mathrm{r}_{\mathrm{M}}, \mathrm{t}\right)=1-\left(1-\varepsilon_{0}\right)\left[\mathrm{C}\left(\mathrm{r}_{\mathrm{M}}, \mathrm{t}\right)\right]^{3} \\
\mathrm{R}_{\mathrm{L}}=6 \mathrm{R}_{\mathrm{C}} \mathrm{L} \quad \mathrm{L}=1,2 \ldots \mathrm{N}
\end{gathered}
$$

In fragmentation region ${ }^{9}$

$$
\varepsilon\left(\mathrm{r}_{\mathrm{M}}, \mathrm{t}\right)=\varepsilon^{*}
$$

$\mathrm{R}_{\mathrm{L}}=\left\{\frac{\left(1-\varepsilon_{0}\right)}{\left(1-\varepsilon^{*}\right)} \times\left[6 \mathrm{C}_{\mathrm{L}}(\mathrm{t}) \mathrm{R}_{\mathrm{C}}\right]^{3} \times\left[\mathrm{L}^{3}-(\mathrm{L}-1)^{3}\right]+\mathrm{R}_{\mathrm{L}-1}^{3}\right\}^{\frac{1}{3}}$ 
where $C_{L}(t)$ denotes time-dependent dimensionless growth factor for a microparticle in layer $\mathrm{L} ; \mathrm{R}\left(\mathrm{r}_{\mathrm{M}}, \mathrm{t}\right)$ is defined as a function of the layer number and macroparticle radius and can be given by Estenoz and Chiovetta'

$$
R_{p}\left(r_{M}, t\right)=\frac{6^{3}\left(1-\varepsilon_{0}\right)\left[L^{3}-(L-1)^{3}\right]}{\frac{4 \pi}{3}\left[R_{L}(t)^{3}-R_{L-1}(t)^{3}\right.} \times 4 \pi R_{C}^{2} k(T) M_{C}\left(r_{M}, t\right)
$$

$\mathrm{k}(\mathrm{T})$ can be calculated as follow ${ }^{9}$ :

$$
\mathrm{k}(\mathrm{T})=\mathrm{k}\left(\mathrm{T}_{0}\right) \exp \left\{\frac{\mathrm{E}}{\mathrm{R}_{\mathrm{g}}}\left[\frac{1}{\mathrm{~T}_{0}}-\frac{1}{\mathrm{~T}\left(\mathrm{r}_{\mathrm{M}}, \mathrm{t}\right)}\right]\right\}
$$

Eqns. 1-17 is the single particle model established for the slurry ethylene polymerization catalyzed with a silicasupported metallocene catalyst in this paper.

Parameter values: In the simulation process, the practical conclusions will depend on the range of parameter values. For the slurry phase polymerization of ethylene catalyzed by supposed metallocene catalyst, these values are tabulated in Table-1 $1^{6,8-10,20}$

TABLE-1

THERMAL, PHYSICAL, MASS TRANSFER PROPERTIES AND RELEVANT PARAMETER VALUES FOR THE SLURRY ETHYLENE POLYMERIZATION

\begin{tabular}{cccc}
\hline Parameters & Value & Parameters & Value \\
\hline $\mathrm{h}\left(\mathrm{cal} / \mathrm{m}^{2} \mathrm{~s}\right)$ & 404 & $\mathrm{M}_{\mathrm{B}}\left(\mathrm{mol} / \mathrm{m}^{3}\right)$ & 155 \\
$\mathrm{D}_{1}\left(\mathrm{~m}^{2} / \mathrm{s}\right)$ & $5.0 \times 10^{-9}$ & $\rho_{\mathrm{p}}\left(\mathrm{g} / \mathrm{m}^{3}\right)$ & $9 \times 10^{5}$ \\
$\mathrm{R}_{\mathrm{C}}(\mathrm{m})$ & $6 \times 10^{-9}$ & $\mathrm{k}(\mathrm{m} / \mathrm{s})$ & $1.2 \times 10^{-6}$ \\
$\mathrm{R}_{\mathrm{m}}(\mathrm{m})$ & $4.5 \times 10^{-5}$ & $\mathrm{E}(\mathrm{cal} / \mathrm{mol})$ & 13500 \\
$\mathrm{~T}(\mathrm{~K})$ & 323 & $\mathrm{X}_{\mathrm{D}}^{20}$ & 0.04 \\
$\mathrm{D}_{\mathrm{S}}\left(\mathrm{m}^{2} / \mathrm{s}\right)$ & $3.33 \times 10^{-10}$ & $\varepsilon_{0}$ & 0.825 \\
$\mathrm{~m}$ & 1250 & $\mathrm{C}_{0}$ & 1.5 \\
$\varepsilon^{*}$ & 0.4 & $\mathrm{~K}_{\mathrm{M}}(\mathrm{cal} / \mathrm{m} \mathrm{s})$ & 0.47 \\
$\mathrm{P}(\mathrm{atm})$ & 1.7 & - & - \\
\hline
\end{tabular}

Simulations and discussion: By the previous research results $^{6-17}$, the heat-transfer can be neglected. So the temperature in the macroparticle is almost the same as that in the reaction. The kinetics constant can be used in all the macroparticle from fitting to the whole particle.

Ethylene monomer concentration distribution: The monomer concentration of profiles at different time is shown in Fig. 2. It can be seen clearly that the curves are typical of a diffusion and reaction system in term of shape. The reaction is controlled by kinetic and diffusion from time to time as shown in the Fig. 2. A surprising phenomenon which can be observed is that the concentration at $100 \mathrm{~s}$ is lower than that $10 \mathrm{~s}$. It could be explained by catalyst fragmentation. The polymerization is controlled by reaction and diffusion at the beginning of reaction and the monomer concentration increases at this moment. As polymerization reaction goes on, the polymer deposits on pores of macroparticles as a barrier, the outer monomer cannot cross through the pore of catalyst and therefore cannot react on active sites of catalyst; the polymerization is controlled by diffusion. After $200 \mathrm{~s}$, catalyst breaks up shell by shell into a large number of smaller microparticles which are encapsulated by polymer chain, mass transfer

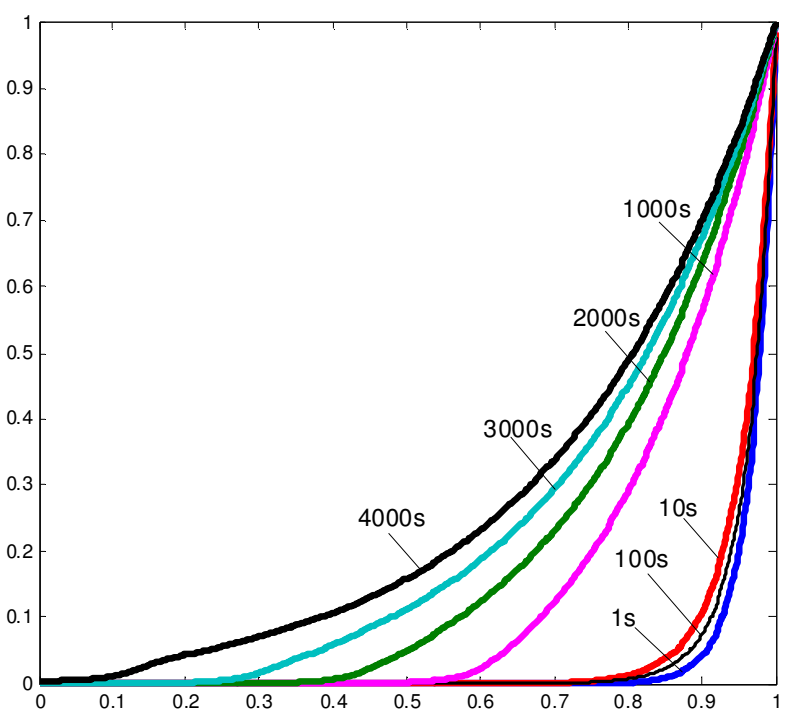

Fig. 2. Ethylene monomer concentration of marcopaticle versus time

resistance would decrease and new active catalyst sites would release; thus, reaction changes from diffusion control to combination of diffusion and kinetics (Fig. 2).

The ethylene monomer concentration at various layers of macropaticle versus time is shown in Fig. 3. It can be seen clearly that the monomer concentration in the outer layer of the macroparticle is almost constant, but the inner layer is almost zero. The probable reason is great mass transfer resistance of the polymerization; it is caused by the polymer deposit on pores of particles, therefore the monomer cannot pass through pores where the polymerization takes place.

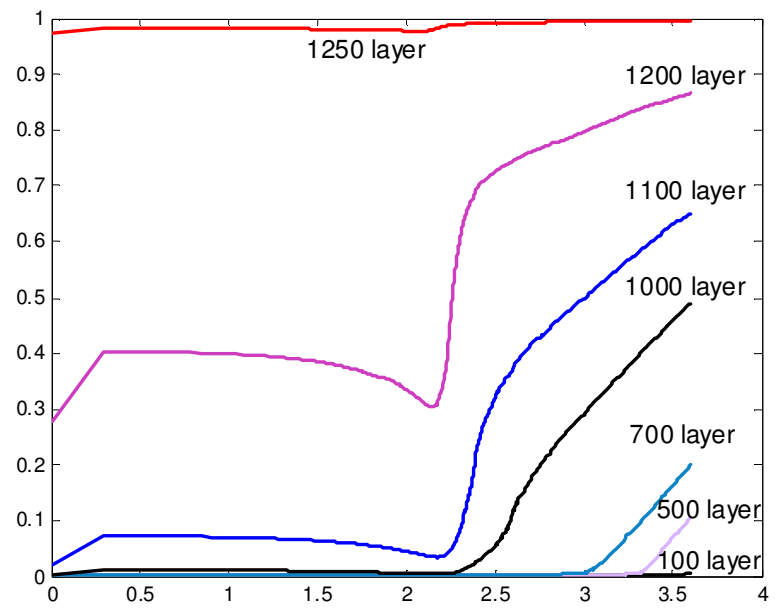

Fig. 3. Ethylene monomer concentration at various layers of macroparticle versus time

Evolution of growth factor and porosity: The microparticle growth factor versus particle radius at various layers is shown in Fig. 4 and porosity versus the macroparticle radius under reaction time is shown in Fig. 5. From Fig. 2, it can be seen that the growth factor increase with time and the growth factor of microparticle in outer layer become slower than in inner layer. The probable reason is the great mass transfer resistance during the early polymerization. Porosity in the particle decrease till it is constant as shown in Fig. 3. 


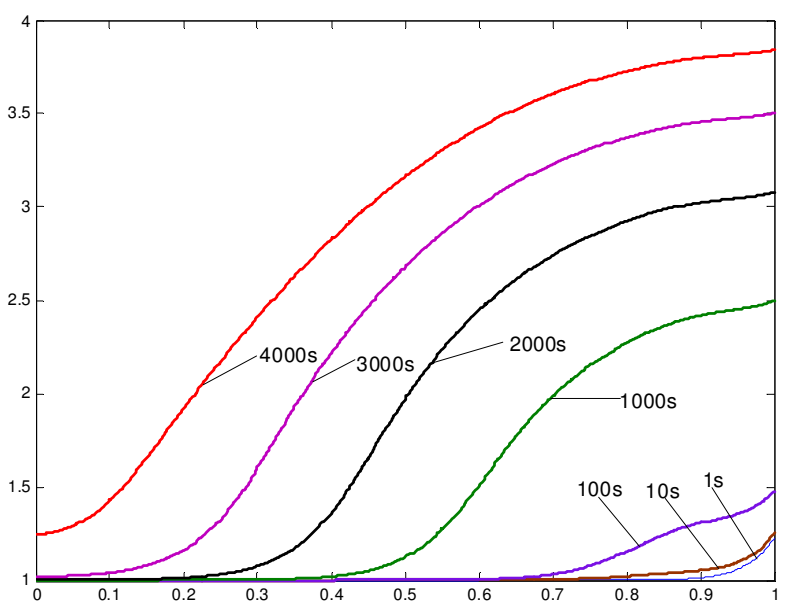

Fig. 4. Microparticle growth factor versus particle radius at various layers

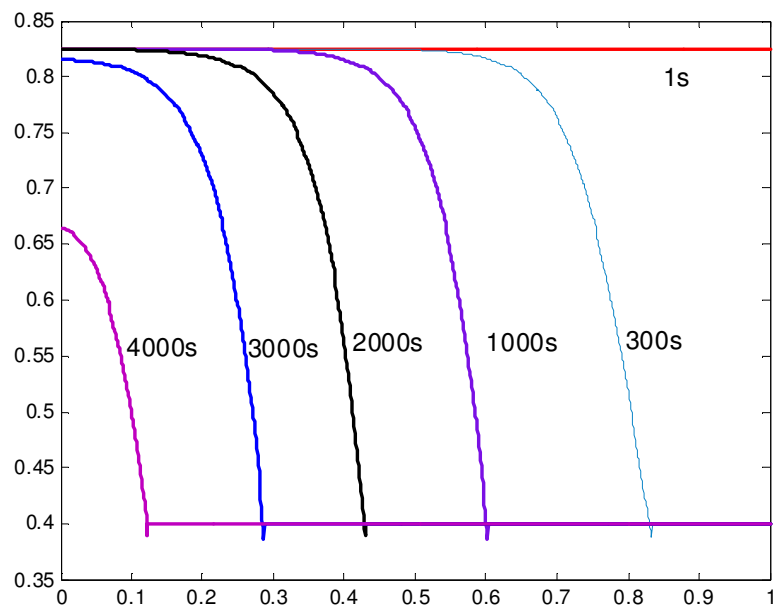

Fig. 5. Porosity versus macroparticle radius under reaction time

Polymerization rate of ethylene: Effect of reaction time on polymerization rate of ethylene is shown in Fig. 6. It can be seen clearly that the polymerization rate of ethylene reaches maximum point in very short time and it decreases rapidly. As it reaches a minimum point, it increases again. The main probable reason is that the catalyst is activated at the moment and the polymerization rate increases. As polymerization goes on, the pore in the particle is blocked by polyethylene, thus the monomer cannot pass the pore and reach the surface of microparticle where the reaction takes place, the reaction rate decreases. When catalysts break up into a large number of smaller microparticles, which are encapsulated by polymer chain at $200 \mathrm{~s}$ of the polymerization time, the mass transfer resistance decreases; thus the polymerization rate increases. As catalyst collapses from surface to center shell by shell, the reaction rate increases constantly as shown in Fig. 6.

\section{Conclusion}

(1) A modified multigrain model, which is investigated the slurry ethylene polymerization catalyzed with a silicasupported metallocene catalyst, is developed. The results show mass transfer resistance has great effect on the polymerization during the early period of the polymerization.

(2) The catalyst fragmentation in the polymerization process is studied. The catalyst fragmentation takes place shell

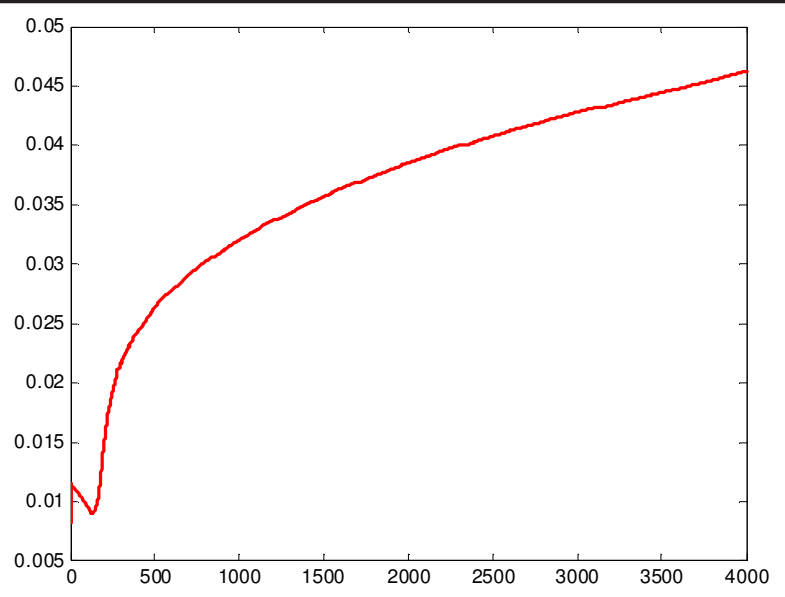

Fig. 6. Effect of reaction time on polymerization rate of ethylene

by shell from the surface to the center after $200 \mathrm{~s}$ in polymerization time.

(3) The slurry ethylene polymerization process is analyzed based on longitudinal (reaction time) and transverse (macroparticle's radius).

(4) The evolution of porosity and variant growth factor are investigated. The results show that the mass transfer resistances have great effect on the reaction.

\section{Nomenclatures}

C : Growth factor

$C_{L}$ : Dimensionless growth growth factor for a microparticle in layer $\mathrm{L}$

$c_{p}:$ Specific heat, $\mathrm{kJ} \mathrm{g}^{-1} \mathrm{~K}^{-1}$

$\mathrm{D}_{1}$ : Monomer diffusion coefficient in the macroparticle, $\mathrm{m}^{2} \mathrm{~s}^{-1}$

$\mathrm{D}_{\mathrm{s}}$ : Monomer diffusion coefficient in the microparticle, $\mathrm{m}^{2} \mathrm{~s}^{-1}$

E : Activation energy, $\mathrm{kJ} \mathrm{mol}^{-1}$

$\mathrm{h}$ : Thermal transfer coefficient in the external film, $\mathrm{kJ} \mathrm{m}^{-2}$ $\mathrm{s}^{-1} \mathrm{~K}^{-1}$

$\mathrm{k} \quad$ : Superficial kinetic constant, $\mathrm{m} \mathrm{s}^{-1}$

$\mathrm{k}_{\mathrm{e}}$ : Macroparticle thermal conductivity, $\mathrm{kJ} \mathrm{m}^{-1} \mathrm{~s}^{-1}$

$\mathrm{k}_{\mathrm{s}}$ : Gas transfer coefficient in the external film, $\mathrm{m}^{2} \mathrm{~s}^{-1}$

L : Macroparticle-layer number

$\mathrm{M}_{\mathrm{B}}$ : Ethylene concentration in bulk, $\mathrm{mol} \mathrm{m} \mathrm{m}^{-3}$

$\mathrm{M}_{\mathrm{c}}$ : Monomer concentration on the surface of catalyst, $\mathrm{mol} \mathrm{m}^{-3}$

$\mathrm{M}_{\mathrm{n}}$ : Ethylene molecular weight, $\mathrm{g} \mathrm{mol}^{-1}$

$\mathrm{M}$ : Monomer concentration, $\mathrm{mol} \mathrm{m}^{-3}$

$\mathrm{m}$ : Total layer of macroparticle

$\mathrm{N}_{2}$ : Number of microspheres in layer $\mathrm{L}$

$\mathrm{P}$ : Pressure, bar

$\mathrm{r}_{1}$ : Variable along macroparticle radius, $\mathrm{m}$

$r_{c}$ : Variable along microparticle radius, $m$

$\mathrm{R}_{\mathrm{L}}$ : External radius of layer $\mathrm{L}, \mathrm{m}$

$\mathrm{R}_{\mathrm{M}}$ : Radius of macroparticle, $\mathrm{m}$

$R_{p}$ : Polymerization rate of ethylene, mol m $\mathrm{m}^{-3} \mathrm{~s}^{-1}$

$\mathrm{R}_{\mathrm{c}}$ : Radius of microparticle, $\mathrm{m}$

$\mathrm{T}$ : Temperature, $\mathrm{K}$

$\mathrm{T}_{\mathrm{B}}$ : Temperature of the bulk fluid phase, $\mathrm{K}$

$\mathrm{t}$ : time, $\mathrm{s}$

$X_{D}$ : Ratio between the monomer diffusion constant in the macroparticle before and after fragmentation 


\section{Greek letters}

$\alpha$ : Amorphous-phase volume fraction of the polymer layer

$\beta$ : Thiele modulus

$\varepsilon \quad:$ Macroparticle porosity

$\varepsilon_{0}$ : Initial porosity of the macroparticle

$\varepsilon^{*}$ : Final porosity of the macroparticle

$\rho_{\mathrm{p}}:$ Polymer density $\left(\mathrm{g} \mathrm{cm}^{-3}\right)$

$\rho_{M}:$ Macroparticle density $\left(\mathrm{g} \mathrm{cm}^{-3}\right)$

$\xi \quad$ : Radius of unfragmentation macroparticle (m)

$\Delta \mathrm{H}:$ Heat of polymerization $\left(\mathrm{kJ} \mathrm{mol}^{-1}\right)$

\section{ACKNOWLEDGEMENTS}

The authors are grateful to the financial support from Department of Science and Technology of Jiangsu Province (BK2010198), Changzhou Municipal Science and Technology Bureau (CS20100004), State Key Laboratory of Green Chemical Synthesis Technology (GCTKF 2010013) and Natural Science Preliminary Research Foundation of Southeast University (XJ0619245).

\section{REFERENCES}

1. T.F. McKenna and J.B.P. Soares, Chem. Eng. Sci., 56, 3931 (2001).

2. J.W. Begley, J. Polym. Sci. A, 4, 319 (1966).

3. W.R. Schmeal and J.R. Street, AIChE J., 17, 1188 (1971).

4. L. Böhm, Chem. Ing. Technol., 56, 674 (1984) (in German).

5. E.J. Nagel, V.A. Krillov and W.H. Ray, Ind. Eng. Chem. Prod. Res. Dev., 19, 372 (1980).

6. F. Bonini, J. Polym. Sci. A: Polym. Chem., 33, 2393 (1995).

7. A. Alexiadis, C. Andes, D. Ferrari, F. Korber, K. Hauschild, M. Bochmann and G. Fink, Macromol. Mater. Eng., 289, 457 (2004).

8. A. Alexiadis and C. Andes, Appl. Math. Model, 32, 99 (2008).

9. D.A. Estenoz and M.G. Chiovetta, J. Appl. Polym. Sci., 81, 285 (2001).

10. G. Fink, B. Steinmetz, J. Zechlin, C. Przybyla and B. Tesche, Chem. Rev., 100, 1377 (2000).

11. A. Yiagopoulos, H. Yiannoulakis, V. Dimos and C. Kiparissides, Chem. Eng. Sci., 56, 3979 (2001).

12. S. Floyd, T. Herskanen, T.W. Taylor, G.E. Mann and W.H. Ray, J. Appl. Polym. Sci., 33, 1021 (1987).

13. P. Sarkar and S.K. Gupta, Polymer, 32, 2842 (1991).

14. P. Sarkar and S.K. Gupta, Polymer, 33, 1477 (1992).

15. U.P. Veera and G. Weickert, AIChE J., 48, 1062 (2002).

16. T.F. McKenna and J.B.P. Soares, J. Appl. Polym. Sci., 63, 315 (1997).

17. M.A. Ferrero and M.G. Chiovetta, Polym. Eng. Sci., 27, 1436 (1987).

18. A. Hutchinson, J. Appl. Polym. Sci., 44, 1389 (1992).

19. F. Floyd, J. Appl. Polym. Sci., 32, 2935 (1986).

20. S. Knoke, F. Korber, G. Fink and B. Tesche, Macromol. Chem. Phys., 204, 607 (2003). 